\title{
Prevalencia del riesgo de alteraciones en el desarrollo neuroconductual en una población de lactantes de México en 2012
}

\author{
Prevalence of the risk of alterations in neurobehavioral development in a \\ population of lactating children in Mexico in 2012
}

\begin{abstract}
Resumen
El objetivo de este estudio fue determinar la prevalencia del riesgo para alteraciones del desarrollo de lactantes de una región del sureste de México, utilizando el instrumento de valoración neuroconductual del desarrollo del lactante VANEDELA. Los niños identificados fueron aptos para recibir estimulación temprana o rehabilitación. Se estudiaron 118 lactantes con edades de 1 a 24 meses, con un promedio de 6,4 meses, con riesgo leve para alteración en un $34 \%$, riesgo alto en un $7 \%$ de los lactantes y el $59 \%$ desarrollo normal. En la valoración de conductas del desarrollo, la mayor prevalencia de afectación se presentó en áreas de los sistemas motor fino $(27,3 \%)$ y cognoscitivo, lenguaje en un $25 \%$ en todas las etapas. El $12 \%$ de lactantes de 4 meses presentó reacciones del desarrollo ausentes y un $6 \%$ de la muestra tuvo signos de alarma, razón por la que fueron remitidos para valoración en el laboratorio de rehabilitación. La prueba VANEDELA fue suficientemente precisa para determinar el riesgo de alteraciones del desarrollo neuroconductual, siendo un test tamizaje utilizado en población aparentemente sana y para sugerir programas integrales de atención temprana o fortalecer los existentes.
\end{abstract}

\section{Palabras clave}

Desarrollo infantil, evaluación, lactante, prevalencia.

\begin{abstract}
The aim of this study was to determine the prevalence of risk for disorders of neurobehavioral development of lactating children living in the southeastern region of Mexico, through the use of the test of lactating children neurobehavioral development VANEDELA. The identified children were able to receive early stimulation or rehabilitation. A total of 118 infants aged 1 to 24 months were studied, with an average of 6.4 months, with a slight risk for alteration in 34\%, high risk in $7 \%$ of infants and $59 \%$ normal development. In the assessment of developmental behaviors, the highest prevalence of involvement appeared in areas of fine motor systems (27.3\%) and cognitive systems, language by $25 \%$ in all stages. $12 \%$ of 4 -month-old infants presented absent developmental reactions and $6 \%$ of the sample had signs of alarm, which is why they were referred for assessment in the rehabilitation laboratory. The VANEDELA test was sufficiently precise to determine the risk of alterations in neurobehavioral development, being a screening test used in apparently healthy population and to suggest comprehensive programs of early attention or strengthen existing ones.
\end{abstract}

\section{Keywords}

Infant development, evaluation, infant, prevalence.

\author{
Livet Rocío Cristancho \\ González \\ <lcristancho968@puce.edu.ec> \\ Pontificia Universidad Católica del \\ Ecuador. Ecuador
}

\section{Enrique Núñez Lara}

Universidad Autónoma del Carmen. México

\section{Martha Patricia Kent Sulú \\ Universidad Autónoma del Carmen.} México

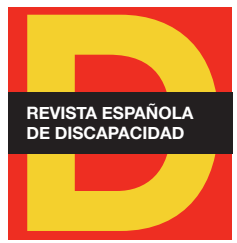

Para citar:

Cristancho, L. R. et al. (2018):

"Prevalencia del riesgo de alteraciones en el desarrollo neuroconductual en una población de lactantes de México en 2012". Revista Española de Discapacidad, 6 (l): 241-255.

Doi: <https://doi.org/10.5569/23405104.06.01.12>

Fecha de recepción: 01-05-2017 Fecha de aceptación: 09-03-2018

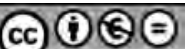




\section{Introducción ${ }^{1}$}

El término 'desarrollo' ha sido definido desde los postulados de Darwin (Salgado, 2007) como el proceso progresivo caracterizado por el incremento de funciones y su mayor coordinación; se refiere a transformaciones globales que conducen a la adaptación. La evolución adecuada del desarrollo no se realiza rígida ni estereotipada, existen variaciones individuales dependiendo del proceso de maduración del sistema nervioso central (SNC) y de la influencia del macro y microambiente, que facilitan o no el pleno desarrollo del niño (Schapira, 2007). Cualquier perturbación de las funciones del SNC es signo trascendente de una disfunción (Iceta y Yoldi, 2002), por lo cual es necesario valorar adecuadamente el desarrollo psicomotor de los niños para detectar tempranamente la disfunción.

La supervisión del desarrollo psicomotor abarca un campo ampliamente estudiado. Las recomendaciones para la valoración del desarrollo muestran la necesidad de contar con referentes adecuados en aspectos conductuales para diferentes momentos del desarrollo. Para esto se cuenta con un grupo pruebas de tamizaje que establecen la relación de las conductas del desarrollo que experimenta el niño, comparándolas con lo esperado para la edad (Bolaños et al., 2006; González et al., 2013; Salazar et al., 2006; Sánchez et al., 2014; Fernández Carrocera et al., 1999) y buscan diferenciar sujetos normales de sujetos con alteración, a escala internacional. Algunas son el test de Denver II, el Clinical Adaptive Test/Clinical Linguistic Auditory Milestone Sacale (CAT/CLAMS), Ages \& Stages Questonaires (ASQ) (Sánchez et al., 2014). En México se cuenta con la Prueba de Valoración Neuroconductual del Desarrollo del Lactante (VANEDELA) y el Perfil de Conductas del Desarrollo. Estos dos últimos están adaptados para la población latina y se han utilizado en numerosos estudios que validan su aplicación (Alvarado et al., 2013; Bolaños et al., 2006; Sánchez et al., 2014).

Para el presente estudio se utilizó la prueba VANEDELA, ya que tiene aplicación importante en Latinoamérica en virtud de su adecuación a los patrones culturales de países latinos, fue validada y probada con población mexicana. Adicionalmente, se considera una prueba de sencilla aplicación, no requiere para su uso de una gran infraestructura y permite entrenar a la madre o al cuidador del menor para que sean valoradas las conductas del lactante de acuerdo con las etapas del desarrollo.

En México, según pruebas de tamizaje se reportan prevalencias de 14,4 al $20 \%$ de riesgo de alteraciones del neurodesarrollo (Alvarado et al., 2013). Estas comúnmente son detectadas durante la consulta de pediatría a manera de filtro, es decir, no por la práctica rutinaria de una prueba tamiz para la medición del desarrollo, un alto número de casos transitan a la edad preescolar con las dificultades que van a interferir con su desempeño escolar (Alvarado et al., 2013). Así mismo, el $2 \%$ de los nacimientos al año están en riesgo de presentar algún tipo de alteración o discapacidad de tipo motriz (INEGI, 2010) debido a la edad de la madre y a las condiciones sociales y económicas prevalecientes en este sentido en el sureste de México, ya que el $35 \%$ de los nacimientos al año se dan en madres menores de 18 años (Instituto Nacional de Salud Pública, 2012).

1. Un agradecimiento especial a los estudiantes de la Licenciatura en Terapia Física, tercer semestre, de la Universidad Autónoma del Carmen (Ciudad del Carmen, México) que participaron en la realización de este estudio, así como al Hospital General Doctora María del Socorro Quiroga Aguilar y a la guardería Baby Delfín de Ciudad del Carmen, por su apoyo durante todo el proceso de la investigación. Especialmente, agradecimiento a los padres que permitieron la valoración de los niños e hicieron parte del estudio. 
La inclusión de pruebas tamiz para la detección rápida de trastornos del desarrollo puede ser una práctica rutinaria en servicios de rehabilitación o estimulación temprana. El presente estudio contribuye a mostrar la importancia de la detección a través de instrumentos validos de valoración o tamizaje (Sánchez et al., 2014; Alvarado et al., 2013; Bolaños et al., 2015; Pelayo et al., 2015), con el fin de que los resultados obtenidos puedan servir de base para la estructuración de futuros programas de atención temprana.

\section{Material y Métodos}

Estudio observacional, descriptivo y transversal, se enfocó en la población infantil lactante de 1 a 24 meses de edad, con una muestra aleatoria de 145 niños de los servicios de salud del Hospital General de Ciudad del Carmen (Campeche, México) de febrero a noviembre de 2012. Se consideraron para la evaluación los lactantes que habiendo nacido en los hospitales, acudieron a controles mensuales de pediatría los días que los estudiantes de terapia física asistían al hospital y algunos lactantes remitidos por el área de hospitalización pediátrica. Sin embargo, solo se incluyeron los menores cuyos padres aceptaron la evaluación y firmaron el consentimiento informado. Así mismo, se incluyeron 26 niños de la guardería de la Universidad Autónoma de Ciudad del Carmen.

Se utilizaron como criterios de exclusión niños que en el momento de la evaluación tuviesen cualquier tipo de enfermedad latente, o sobre los que en pediatría se informara de que no se encontraban en condiciones para responder fielmente a los reactivos, así como niños que asistieron a la consulta con un familiar diferente a sus padres, pues se requería la información del cuidador (a estos niños se les programó una cita posterior). Otro criterio fue el de presentar una discapacidad.

Los criterios de inclusión fueron niños en buen estado de salud, es decir, que estuvieran en la consulta externa de pediatría para control y no para manejo de una situación de enfermedad, por cuanto estos niños se presumía que no responderían adecuadamente a la prueba (sin embargo fueron programados para una cita posterior). Otro criterio fue presentarse con uno de sus padres y tener entre 1 y 24 meses de edad.

Se incluyó como prueba de evaluación VANEDELA, considerando que el objetivo del estudio fue demostrar la prevalencia de riesgo para alteraciones en el desarrollo en un grupo aleatorio de población considerada sana. Sin embargo para casos con un riesgo alto de alteración se remitió al laboratorio de rehabilitación en donde podría ser evaluado con pruebas más específicas de diagnóstico según la edad.

La prueba de tamizaje VANEDELA, en su primera versión, fue diseñada para población mexicana en el periodo de 1983 a 1985 por un grupo de investigadores del Instituto de Neurodesarrollo de la Universidad Autónoma Metropolitana de Xochimilco. Dicho instrumento ha servido en numerosos estudios dirigidos por los autores y actualmente tiene una aplicación importante en Latinoamérica por las características mismas de la prueba, como la facilidad de manejo y su adecuación a los patrones culturales de países latinos. Consta de criterios estandarizados en edades de corte, 1, 4, 8, 12, 18 y 24 meses, con cuatro categorías de evaluación como son la valoración somatométrica, conductas del desarrollo, reacciones del desarrollo y signos de 
alarma. Las calificaciones son nominales de presente o ausente, para una puntuación de 0 a 10, y en porcentajes de 0 a 100, considerando que una calificación menor o igual a $70 \%$ es indicativa de alteración en la conducta del desarrollo. En cuanto a las reacciones del desarrollo se evalúan como presentes o ausentes también, y en los signos de alarma la calificación es el registro del signo que se ha presentado. Después de una calificación global, todo niño que no cumpla con los parámetros del desarrollo para la edad debe ser remitido a atención primaria y aquellos que tengan una calificación igual o menor a 70 deben ser canalizados a una valoración y manejo especializado adecuado a la alteración presente (Chávez et al., 2012).

El instrumento fue la prueba VANEDELA. No puede ser usado como instrumento de diagnóstico para alteraciones del desarrollo, ni permite evaluar fuera de las edades de corte, observa factores relevantes del desarrollo e incluye manifestaciones conductuales que presentan los niños bajo condiciones óptimas. El instrumento consta de cuatro partes:

1. Evaluación somatométrica: ES.

2. Evaluación de conductas del desarrollo: CD.

3. Evaluación de las reacciones del desarrollo: RD.

4. Evaluación de los signos de alteración: SA.

Para la presente investigación se tomaron las variables de sexo, edad del niño y las variables de VANEDELA. Previo a la aplicación de la prueba se realizó un taller dirigido a los profesionales de pediatría, UCI neonatal, enfermería y administrativos del hospital para informar sobre objetivos y procedimientos del estudio, así como las etapas de la evaluación.

El estudio no contó con una nutricionista para la realización de la primera parte de la prueba, por lo tanto se realizó con énfasis en las tres últimas partes. La evaluación de conductas del desarrollo (CD), agrupa áreas como: alimentación, perceptivo auditivo, perceptivo visual, reflejos, postura antigravitatoria, motor grueso, deambulación, coordinación ojo-mano, motor fino, manipulación, emocional social, cognoscitivo, lenguaje expresivo y lenguaje receptivo. Las manifestaciones conductuales se distribuyen en 60 reactivos en total, 10 para cada una de las edades de corte: 1, 4, 8, 12, 18 y 24 meses. La evaluación de los 10 reactivos para cada lactante fue realizada a través de la observación directa del explorador. Cada reactivo contó con dos intentos para ver la respuesta y se calificó como positiva o presente (1) y negativa o ausente (0). La posición para la valoración fue la requerida por el reactivo realizada por un terapeuta encargado del estudio y un estudiante de Terapia Física. Los resultados en las CD son categorizados como: i) desarrollo normal, cuando los 10 indicadores o reactivos fueron positivos, ii) en duda cuando 8 o 9 indicadores son positivos y iii) anormal, cuando 7 o menos indicadores son positivos.

En cuanto a las reacciones del desarrollo (RD), correspondieron a la evaluación de reflejos que son necesarios para el paso de una etapa neuroconductual a otra permitiendo establecer patrones posturales específicos para cada edad de corte. Es así como al mes se valoró el reflejo de enderezamiento óptico laberintico y el enderezamiento de la cabeza sobre el cuerpo; a los 4 meses el reflejo de Landau; a los 8 meses los reflejos de enderezamiento del cuerpo sobre el cuerpo, bilateral y el de reacciones de protección bilateral; a los 12 meses los reflejos de reacciones de protección hacia los lados y las reacciones de protección hacia atrás bilateral; a los 18 meses las reacciones de equilibrio sentado y en cuatro puntos, bilateral, y a los 24 meses 
las reacciones de equilibrio de pie. Cada reflejo contó con dos intentos para su evaluación y se calificó como presente $(\mathrm{P})$, ausente $(\mathrm{A})$ y conducta alterada $(\mathrm{CA})$, lo cual se identifica como signo de alteración.

Posteriormente se identificaron los signos de alarma (SA), que son determinadas conductas que reflejan inmadurez del sistema nervioso central y que están descritas en la prueba teniendo en cuenta los hitos del desarrollo (Sánchez et al., 2015). En el siguiente cuadro se recopilaron algunos signos neurológicos de alarma durante los primeros 24 meses de vida, según la propuesta de Sánchez et al. (2015).



Fuente: elaboración propia basada en Sánchez et al., 2015. 
La observación de los signos de alarma en el estudio se realizó a través de observación directa en las etapas de la evaluación y en todas las áreas del desarrollo como: alimentación, perceptual auditivo-visual, motor, emocional social, cognoscitivo, lenguaje y otros signos como convulsiones, asimetrías y alteraciones en los reflejos (Sánchez et al., 2014). Al final de las tres etapas se califica la ponderación de los resultados de VANEDELA así:

- Normal: si tiene calificación en CD de 10, ninguna conducta refleja ausente y ningún signo de alarma.

- Dudoso: cuando el resultado de las CD es de 8 o 9, una RD ausente y un SA.

- $\quad$ Alterado: cuando el resultado de las CD es de 7 o menos, una RD ausente y más de un SA.

Para las variables obtenidas a partir de los puntajes de la prueba se calcularon medidas de tendencia central: media, valores mínimos y máximos y para las variables con respuestas dicotómica (Sí - No) se calculó el porcentaje de niños por respuesta. Cuando tres indicadores fueron rehusados o inaplicables por que el niño estuviera cansado, dormido, inquieto, irritable o con algún signo de alteración, se postergó la aplicación de la prueba.

Los datos obtenidos con la aplicación de la prueba fueron analizados de forma cualitativa considerando la escala de valoración, calculando el porcentaje de niños que se ubicaron en cada una de las tres categorías de riesgo para alteración del desarrollo neuroconductual. En los casos donde el resultado fue dudoso se realizó un análisis con el fin de ubicar problemas que pudieran haber sido derivados de la aplicación de la prueba o de conductas que hubieran sido calificadas como ausentes sin que lo fueran. Los niños calificados como dudosos se observaron tres meses después de terminarse el estudio, en el laboratorio de rehabilitación de la Universidad Autónoma del Carmen, realizando la misma prueba. No obstante, estos datos no fueron considerados para este artículo, pero sí fueron tenidos en cuenta para atenderlos en el laboratorio.

Así mismo, los datos obtenidos de las variables en conductas del desarrollo y las reacciones del desarrollo se relacionaron entre sí por grupo de edad mediante regresión lineal simple con el fin de establecer parámetros de concordancia entre los dos factores y si los resultados de la calificación mostraban el nivel de desarrollo. Adicionalmente en estos casos se relacionaban los signos de alarma presentes, corroborando el resultado de la valoración.

\section{Resultados}

Se incluyeron 145 lactantes de nacionalidad mexicana, 27 de los cuales no completaron las tres etapas de la valoración, por lo que dichas evaluaciones no fueron consideradas para los resultados, quedando 118 lactantes valorados. La edad promedio de los lactantes fue de 6,4 meses, edades comprendidas entre $1 \mathrm{y}$ 24 meses. La distribución por edades fue la siguiente: 23 lactantes de 1 mes, 28 niños de 4 meses, 21 niños en la etapa de 8 meses, 21 niños de 12 meses, 13 niños de 18 meses y 12 niños de 24 meses. La forma como se repartieron los niños para cada etapa fue aquellos que cumplían más o menos 3 semanas antes 
o después de la edad de corte, así por ejemplo los niños que tuvieron 1 mes y tres semanas se incluyeron en la evaluación de un mes, esto se repitió en las demás edades de corte. El 53,2 \% de los lactantes fueron de sexo femenino y el $46,8 \%$ masculino. Los resultados de la valoración de conductas del desarrollo se muestran en la tabla 2.

En la valoración de las conductas del desarrollo neuroconductual, de manera general se determinó que la menor calificación obtenida fue 3 y la mayor 10. Se identificó que el $7 \%$ de lactantes presentan riesgo alto de alteración, la menor calificación en este rango fue 3 con desviación de retardo en 1,2 meses, es decir, retardo del desarrollo de tipo leve. Los lactantes con calificación menor que 7 en las conductas del desarrollo presentaron afectadas tres o cuatro áreas del desarrollo (tabla 1).

Tabla 1. Distribución del riesgo de alteración en el desarrollo neuroconductual según la prueba VANEDELA por edad

\begin{tabular}{|l|c|c|}
\hline Resultado Evaluación Total & Lactantes & Porcentaje \\
\hline Normal & 68 & $59 \%$ \\
\hline Dudoso & 40 & $34 \%$ \\
\hline Alterado & 10 & $7 \%$ \\
\hline Total & 118 & $100 \%$ \\
\hline
\end{tabular}

Fuente: elaboración propia a partir de la base de datos 'Prevalencia de alteraciones en el desarrollo neuroconductual de lactantes. Sureste de México 2012'.

Al mes, las conductas de desarrollo ausentes con mayor frecuencia fueron 'Realiza contacto visual' y el 'seguimiento visual' en un $8 \%$. 'Sostiene por 3 segundos la cabeza o intenta enderezarla' y 'Flexión de miembros superiores' se mostró ausente en un $4 \%$. En el grafico 1 se observan los resultados de la valoración para la edad.

\section{Grafico 1. Evaluación de conductas del desarrollo (1 mes)}


Reactivos para la edad

Positivo

Neqativo

Fuente: elaboración propia a partir de la base de datos 'Prevalencia de alteraciones en el desarrollo neuroconductual de lactantes. Sureste de México 2012'. 
Para 4 meses de edad, las conductas que se evidenciaron con dificultad fueron las de 'Se apoya en antebrazos y levanta la cabeza' en un $18 \%$ y 'No le molesta la posición' en un $18 \%$ ausente. En la conducta de 'Intenta tocar objetos' también ausente en un $18 \%$ y en la de 'Se lleva a la boca el objeto' en un $11 \%$ ausente. Los resultados de la evaluación de CD para la edad se muestran en el gráfico 2.

\section{Grafico 2. Evaluación de conductas del desarrollo (4 meses)}

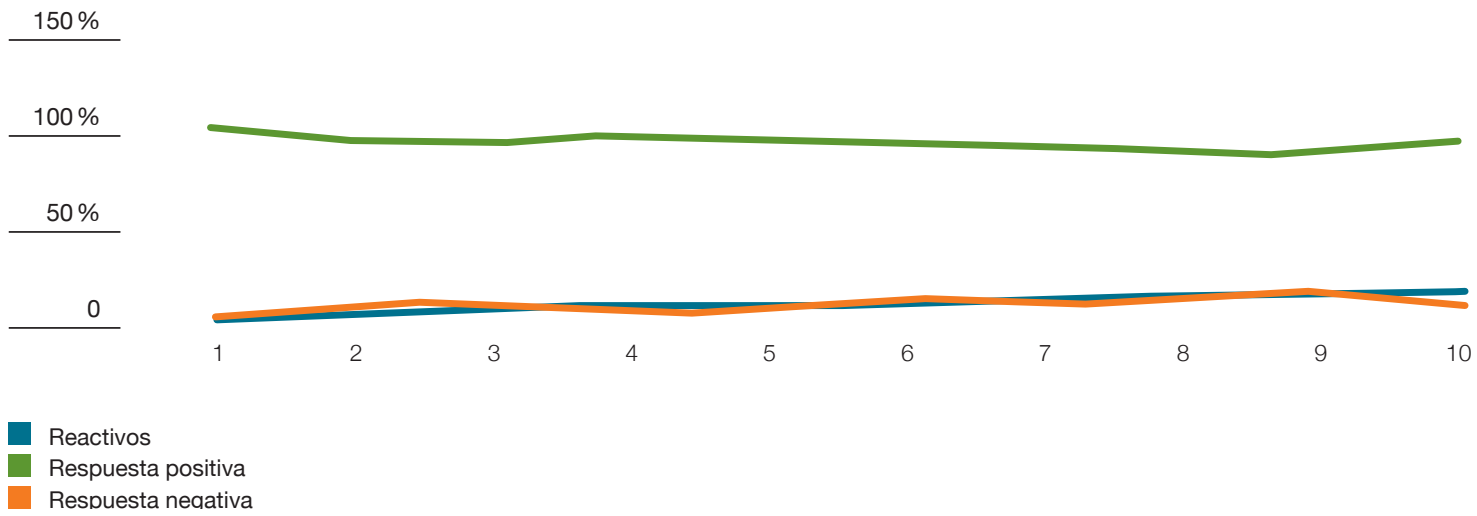

Fuente: elaboración propia a partir de la base de datos 'Prevalencia de alteraciones en el desarrollo neuroconductual de lactantes. Sureste de México 2012'.

A los 8 meses de edad, las actividades ausentes con mayor frecuencia fueron la de 'Se apoya en un brazo para alcanzar un objeto' en un $22 \%$, y 'Encuentra el juguete parcialmente escondido', 'Se apoya en los antebrazos y levanta el tórax', 'Come solo una galleta' y 'Hace caso a su nombre', todas ausentes en un $17 \%$. Los resultados globales se presentan en el grafico 3 .

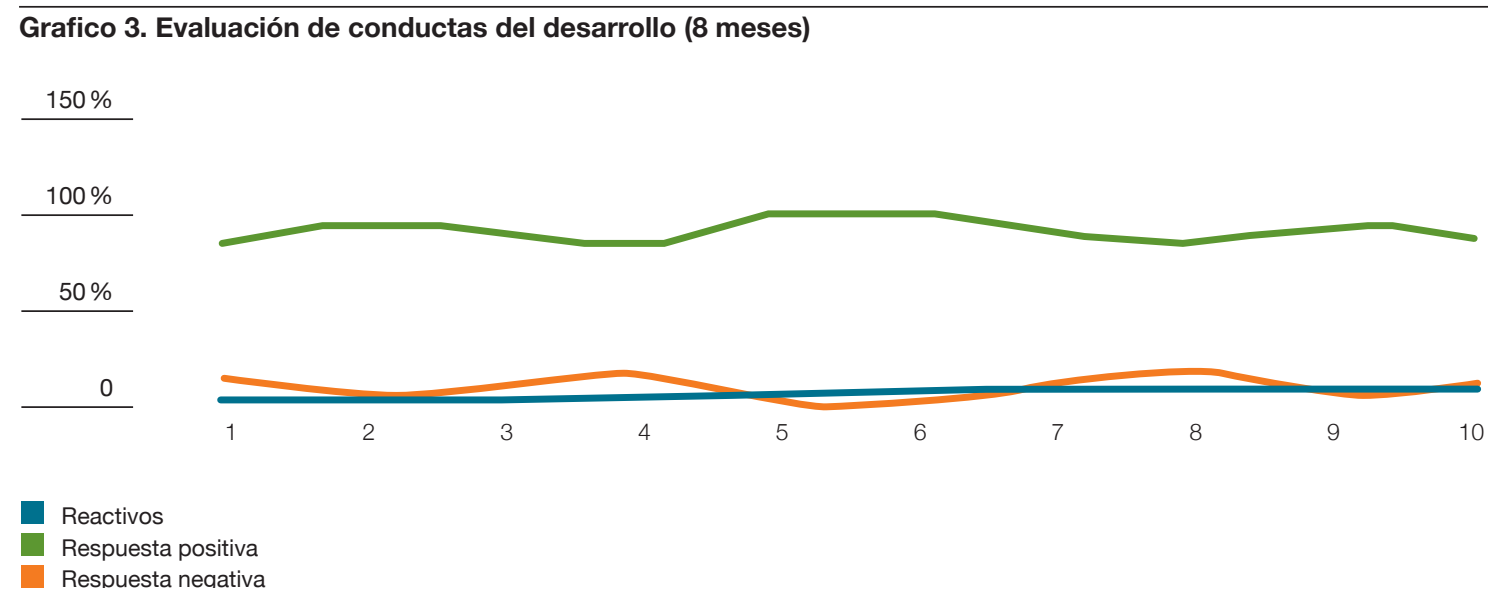

Fuente: elaboración propia a partir de la base de datos 'Prevalencia de alteraciones en el desarrollo neuroconductual de lactantes. Sureste de México 2012'. 
A los 12 meses de edad las conductas más frecuentemente ausentes fueron la de 'Camina bien sostenido de una mano' y 'Se desplaza a gatas, lateral, elefante, sentado', en un 17\%. 'Realiza ordenes sencillas con un gesto', 'Ven dame, no hagas eso', 'Hace monerías con las manos' y 'Bebe solo de una taza', se mostraron ausentes en un $13 \%$. En el siguiente gráfico se muestran los resultados globales de la evaluación de CD.

\section{Grafico 4. Evaluación de conductas del desarrollo (12 meses)}



Fuente: elaboración propia a partir de la base de datos 'Prevalencia de alteraciones en el desarrollo neuroconductual de lactantes. Sureste de México 2012'.

A los 18 meses, 'Dice papa, mamá, más de tres palabras' y 'Saca piedras volteando el frasco', se mostraron ausentes en un $20 \%$ y 'Señala una o más partes de su cuerpo' en un $13 \%$. En el gráfico 5 se muestran los resultados de la evaluación de CD.

\section{Grafico 5. Evaluación de conductas del desarrollo (18 meses)}



Fuente: elaboración propia a partir de la base de datos 'Prevalencia de alteraciones en el desarrollo neuroconductual de lactantes. Sureste de México 2012'. 
Finalmente, a los 24 meses, 'Se llama por su nombre o nene', se mostró ausente en un $25 \%$, y 'Realiza frases de dos palabras' y 'Se trepa en una silla de adulto y para obtener algo' en un $13 \%$ (gráfico 6).

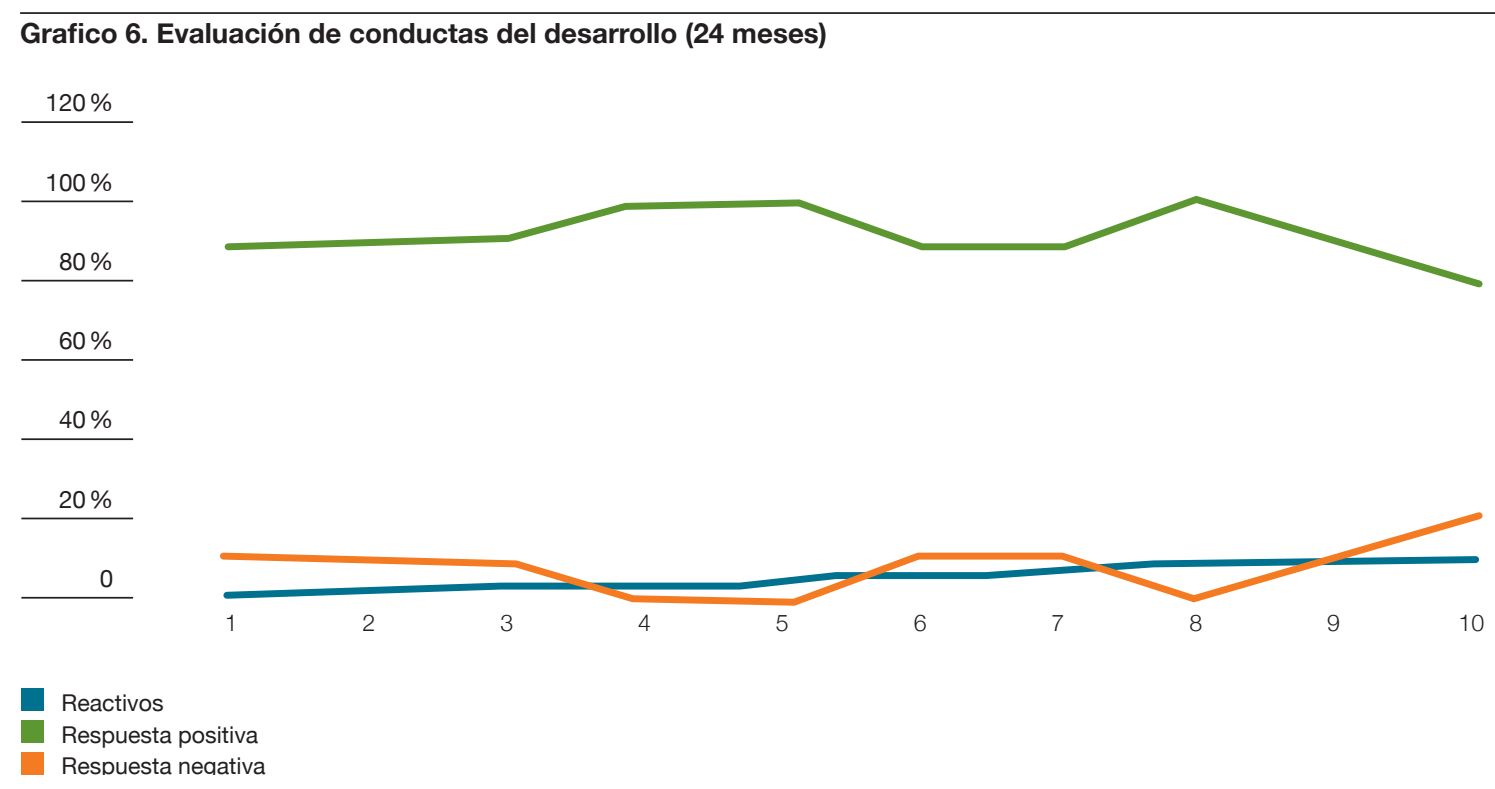

Fuente: elaboración propia a partir de la base de datos 'Prevalencia de alteraciones en el desarrollo neuroconductual de lactantes. Sureste de México 2012'.

Las edades que presentaron mayor cantidad de lactantes con conductas ausentes fueron: a los 24 meses para la conducta de 'Llamarse por su nombre', en un $25 \%$ ausente; a los 8 meses la actividad de 'Se apoya en un brazo para alcanzar un objeto', en un $22 \%$, y a los 12 meses las conductas de decir 'Pa-pá, ma-má inespecífico' y 'Sacar las piedras volteando el frasco', ausentes en un $20 \%$.

Por áreas de desempeño, las conductas con mayor frecuencia ausentes fueron las áreas de motor fino y manipulación, seguido de motor grueso y deambulación, así como postura antigravitatoria, esto para las edades de un mes hasta 12 meses. Para los grupos de 18 y 24 meses se presentaron más ausentes las áreas de lenguaje, motor fino y alimentación. Todos los resultados por edad de evaluación de las conductas del desarrollo se muestran en el tabla 2.

En la valoración de las reacciones en el desarrollo, 20 lactantes tuvieron respuestas ausentes a la prueba, es decir, un $17 \%$ de los lactantes, así como 27 de los niños presentaron signos de alarma, es decir, un $23 \%$ de la muestra. Algunos de los signos que más se repitieron hasta 4 meses fueron el de asimetría derecha o izquierda en una parte de su cuerpo, no fija la mirada y no sostiene la cabeza; para las edades entre 8 y 12 meses, se priva a llorar, no hace caso y es difícil de manejar, y en las edades de 18 y 24 meses algunos signos fueron no explora, no hace caso, no entiende y no habla. 
Tabla 2. Resultado de valoración de conductas del desarrollo según la edad, mediante VANEDELA

\begin{tabular}{|c|c|c|c|}
\hline \multicolumn{2}{|l|}{ Indicadores } & \multirow{2}{*}{$\begin{array}{c}\text { Positivo } \\
1\end{array}$} & \multirow{2}{*}{$\begin{array}{c}\text { Negativo } \\
0\end{array}$} \\
\hline 1 MES & Indicador & & \\
\hline 1. Come sin atragantarse o ponerse morado & 1 & 98,0 & 2,0 \\
\hline 2. Reflejo de presión palmar (ambas manos) & 2 & 100,0 & 0,0 \\
\hline 3. Oye sonidos de la sonaja y detiene o aumenta al movimiento & 3 & 95,9 & 4,1 \\
\hline 4. Contacto visual & 4 & 93,6 & 6,4 \\
\hline 5. Seguimiento visual $90^{\circ}\left(45^{\circ} / 45^{\circ}\right)$ & 5 & 93,5 & 6,5 \\
\hline 6. Sostiene 3 segundos la cabeza o intenta enderezarla & 6 & 95,9 & 4,1 \\
\hline 7. Libera cara & 7 & 98,0 & 2,0 \\
\hline 8. Flexión de los miembros & 8 & 95,7 & 4,3 \\
\hline 9. Llora fuerte cuando está molesto & 9 & 100,0 & 0,0 \\
\hline 10. Se tranquiliza y acurruca al cargarlo & 10 & 100,0 & 0,0 \\
\hline 4 MESES & Indicador & 1 & 0 \\
\hline 1. No rechaza la papilla, succión enérgica & 1 & 100,00 & 0,0 \\
\hline 2. Presión de contacto & 2 & 93,88 & 6,1 \\
\hline 3. Y se lo lleva a la boca & 3 & 93,62 & 6,4 \\
\hline 4. Al jugar platica o se ríe & 4 & 97,96 & 2,0 \\
\hline 5. Seguimiento a 100 & 5 & 97,92 & 2,1 \\
\hline 6. Intenta tocar objetos & 6 & 91,84 & 8,2 \\
\hline 7. Al jalarlo a sentarse la cabeza se alinea al cuerpo & 7 & 93,88 & 6,1 \\
\hline 8. Apoya en antebrazos y levanta la cabeza & 8 & 87,76 & 12,2 \\
\hline 9. No le molesta la posición & 9 & 85,71 & 14,3 \\
\hline 10. Vocaliza espontáneamente o cuando se le habla & 10 & 93,62 & 6,4 \\
\hline 8 MESES & Indicador & 1 & 0 \\
\hline 1. Come una galleta solo & 1 & 86,36 & 13,6 \\
\hline 2. Se mantiene sentado sin ayuda & 2 & 95,65 & 4,3 \\
\hline 3. Toma un objeto en cada mano & 3 & 90,48 & 9,5 \\
\hline 4. Encuentra el juguete parcialmente escondido & 4 & 83,33 & 16,7 \\
\hline 5. Explora con interés la cara de su madre & 5 & 100,00 & 0,0 \\
\hline 6. Al jalarlo a sentarse adelanta la cabeza y estira piernas & 6 & 100,00 & 0,0 \\
\hline 7. Se apoya en las manos y levanta el tórax & 7 & 91,30 & 8,7 \\
\hline 8. Se apoya en un brazo para alcanzar un objeto & 8 & 85,00 & 15,0 \\
\hline 9. Balbuceo ba-ba ta-ta ma-ma & 9 & 95,45 & 4,5 \\
\hline 10. Hace caso a su nombre & 10 & 88,89 & 11,1 \\
\hline 12 MESES & Indicador & 1 & 0 \\
\hline 1. Bebe de una taza & 1 & 90,91 & 9,1 \\
\hline 2. Presión fina & 2 & 96,88 & 3,1 \\
\hline 3. Hace monerías con las manos imitando & 3 & 93,75 & 6,3 \\
\hline 4. Sentado agarra o levanta la pelota & 4 & 100,00 & 0,0 \\
\hline 5. Realiza el juego & 5 & 96,77 & 3,2 \\
\hline 6. Se para agarrándose de los objetos & 6 & 96,88 & 3,1 \\
\hline 7. Se desplaza a gatas, lateral, elefante, sentado & 7 & 90,32 & 9,7 \\
\hline 8. Camina bien sostenido de una mano & 8 & 90,00 & 10,0 \\
\hline 9. Pa-pá, ma-ma inespecífico & 9 & 94,44 & 5,6 \\
\hline 10. Realiza órdenes sencillas con gesto, ven, dame, no hagas eso & 10 & 93,33 & 6,7 \\
\hline
\end{tabular}




\begin{tabular}{|c|c|c|c|}
\hline \multicolumn{2}{|l|}{ Indicadores } & \multirow{2}{*}{$\begin{array}{c}\text { Positivo } \\
1\end{array}$} & \multirow{2}{*}{$\begin{array}{c}\text { Negativo } \\
0\end{array}$} \\
\hline 18 MESES & Indicador & & \\
\hline 1. Come solo con la cuchara aunque derrame & 1 & 100,00 & 0,0 \\
\hline 2. Mete semillas o piedritas en un frasco & 2 & 91,67 & 8,3 \\
\hline 3. Saca las piedras volteando el frasco & 3 & 75,00 & 25,0 \\
\hline 4. Reconoce dos objetos o personas en fotografía & 4 & 81,82 & 18,2 \\
\hline 5. De pie tira la pelota con una o dos manos & 5 & 100,00 & 0,0 \\
\hline 6. Juega entendiendo el juego & 6 & 91,67 & 8,3 \\
\hline 7. Puede acuclillarse y volver a pararse sin ayuda & 7 & 91,67 & 8,3 \\
\hline 8. Camina solo & 8 & 91,67 & 8,3 \\
\hline 9. Dice papá, mama más tres palabras & 9 & 75,00 & 25,0 \\
\hline 10. Señala uno o más partes del cuerpo & 10 & 83,33 & 16,7 \\
\hline 24 MESES & Indicador & 1 & 0 \\
\hline 1. Desenvuelve el dulce o plátano & 1 & 90,00 & 10 \\
\hline 2. Y se lo come & 2 & 90,91 & 9,1 \\
\hline 3. Copia una línea en cualquier dirección & 3 & 90,91 & 9,1 \\
\hline 4. Ayuda por imitación a los quehaceres & 4 & 100,00 & 0,0 \\
\hline 5. Patea la pelota & 5 & 100,00 & 0,0 \\
\hline 6. Se trepa a una silla de adulto & 6 & 90,00 & 10,0 \\
\hline 7. Para obtener algo & 7 & 90,00 & 10,0 \\
\hline 8. Corre & 8 & 100,00 & 0,0 \\
\hline 9. Frase de dos palabras & 9 & 90,00 & 10,0 \\
\hline 10. Se llama por su nombre o nene & 10 & 80,00 & 20,0 \\
\hline
\end{tabular}

Fuente: base de datos estudio de alteraciones del desarrollo neuroconductual en lactantes, VANEDELA, Noviembre de 2011. Ciudad del Carmen, Campeche.

\section{Discusión}

El desarrollo neuroconductual en los lactantes precisa un seguimiento cuidadoso durante los dos primeros años de vida. Esto permite valorar el desempeño del sistema nervioso central en aspectos de la percepción, la cognición y el desarrollo motor, que están profundamente influenciados por la interacción que se brinde con el medio ambiente o del macro y microambiente (Bolaños et al., 2015).

El presente estudio presentó 10 lactantes $(0,07)$ que obtuvieron calificación menor o igual a 7 en la prueba VANEDELA, mostrando la presencia de un retardo de 1,2 meses de conductas del desarrollo, es decir, se reportaron más casos de alteración leve que de otro tipo. Este hallazgo coincide con Alvarado et al. (2013), quienes investigaron en una clínica del Distrito Federal Mexicano a 293 lactantes, utilizando la misma prueba y encontraron riesgo alto para presencia de alteración en un $18 \%$ y riesgo leve en un $30 \%$. Refiere el autor que todos los niños que presentan riesgo de alteración podrían pasar desapercibidos, incluso hasta llegar a la edad preescolar. 
Entre las áreas del desarrollo más afectadas en la valoración de CD para el primer mes destaca la percepción visual en 23 niños (0,08). Algunos estudios han demostrado que a las dos semanas de nacido, el bebé tiene la capacidad de fijar la mirada y a las 4 semanas se instaura la capacidad de realizar seguimiento visual en un angulo de $90^{\circ}$ (Iceta y Yoldi, 2002).

A los 4 meses, la conductas ausentes en mayor porcentaje fueron actividades relacionadas con la postura antigravitatoria $(0,18)$, coincidentes con la demanda del desarrollo de acuerdo al período. Esto quiere decir que en la medida que el lactante crece y se da el desarrollo del sistema nervioso central, aumenta la demanda en el desarrollo o se hace más compleja y por tanto las anomalías también (Salazar et al., 2006). Según Gesell (Alvarado et al., 2013), a los tres meses, en posición prona, el niño puede mantener su peso sin dificultad y descansa en codos y antebrazos, lo que puede sugerir la inexperiencia de los padres en la estimulación y colaboración en el desarrollo motor de los bebes, ya que al observar que el niño se incomoda en la posición prona, prefieren mantenerlo mucha parte del día en la posición boca arriba.

En concordancia con la idea de la progresión compleja en las demandas del desarrollo, a los 8 meses la muestra tuvo entre las conductas ausentes la postura antigravitatoria $(0,22)$ y las areas de motricidad gruesa y deambulación $(0,17)$. Dichas actividades son parte de la secuencia evolutiva en el desarrollo, lo que sugiere que pueden haber traído un rezago de meses anteriores como lo reportan otros estudios (Iceta y Yoldi, 2002).

Las áreas de alimentación y lenguaje para los niños de 8 y 12 meses fueron las más afectadas $(0,17)$. Dichas conductas involucran la habilidad motora y la postura corporal en la alimentación y muestran deficiencias en la integración de las actividades propias de la vida del niño, pues se observó muy arraigado en las madres el patrón cultural en el uso de mamila o el biberón con chupo, por lo que posiblemente el niño no se ve forzado a desarrollarlas.

En cuanto al área de lenguaje, relacionado con la inteligencia o la cognición se encontraron similitudes con el estudio de Alvarado et al. (2013) a los 8 meses $(0,17)$, a los 12 meses $(0,13)$, a los 18 meses $(0,20)$ y a los 24 meses $(0,25)$ con calificación ausente. Este proceso se desarrolla mediante la interacción del niño con su medio, comenzando desde los 4 meses con el balbuceo, la expresión de tres palabras distintas a los 13 meses y combinación de 2 o más palabras a los 21 meses (Schapira, 2007), por lo que este aspecto debe ser analizado desde la perspectiva de la inteligencia. Así mismo, en relación con el lenguaje expresivo, entre 9 y 2 meses se producen las primeras palabras para denominar de forma inespecífica. Posteriormente usa palabras aprendidas en su ambiente alrededor del primer año. Esta habilidad que se relaciona con el desarrollo cognitivo y la interacción con el medio no se vio en los niños de 18 meses a la hora de necesitar unir dos o más palabras para realizar una demanda, por lo que se considera baja la estimulación de los padres en cuanto a la expresión oral de los niños.

La valoración de las reacciones del desarrollo en los lactantes evaluados mostró que en los primeros meses de vida los lactantes presentaban ausentes reflejos como el óptico laberíntico $(0,12)$, quienes no lograron enderezar la cabeza ni alinearla al tronco en posición prono, lo cual requirió continuar vigilando el desarrollo en el laboratorio de rehabilitación y valoración con neurología pediátrica.

En el grupo de 18 meses $(0,12)$ de la muestra, los participantes presentaron ausente la reacción de equilibrio en cuatro puntos, lo que se observaba en un desbalance corporal en la marcha y asimetría en los dos lados 
del cuerpo. En todos los niños fueron observados y registrados los signos de alarma, encontrando significación en las áreas motora, como un desbalance, hipertono o hipotono en algún grado, o asimetrías motoras $(0,65)$ y signos en las áreas emocional social $(0,07)$, como llanto ronco o llanto fuerte y difícil de calmar. Todos fueron remitidos para valoración, atención y vigilancia en el laboratorio de rehabilitación.

En general, los resultados de la valoración del desarrollo neuroconductual en todas sus etapas fue de gran impacto. La prueba VANEDELA fue dada a conocer a los pediatras de los servicios de consulta externa y hospitalización donde se obtuvo la muestra, así como en la guardería, lo que permitió difundir la importancia de tener una prueba tamiz en la consulta como parte del protocolo para la atención en los programas de crecimiento y desarrollo y resaltar la importancia en la detección de lactantes con alteraciones para ser atendido.

\section{Conclusiones y recomendaciones}

Los resultados obtenidos por la aplicación de la prueba VANEDELA fueron suficientemente confiables, permitiendo evaluar el desarrollo en diferentes áreas de forma cualitativa y la utilización de programas de estimulación temprana o de la atención especializada en algunos casos.

La aplicación de la prueba en población lactante del sureste de México mostró que existe un porcentaje importante de niños con riesgo de alteración. La prueba fue suficientemente precisa para detectar el riesgo de alteraciones del desarrollo neuroconductual. Así mismo, se evidenciaron factores de riesgo después del nacimiento, considerando algunos factores culturales y del entorno.

Las áreas con mayor afectación en el desarrollo neuroconductual de los lactantes evaluados, fueron principalmente motora, cognitiva y lenguaje, sin embargo en cada etapa del desarrollo, dependiendo de la complejidad de las demandas neurológicas, fueron apareciendo progresivamente otras áreas involucradas. Esto permitió determinar que cuanto más temprano se aplique la valoración del desarrollo neuroconductual, más rápido serán influenciadas las áreas subsiguientes y más complejas en el desarrollo.

Se hace necesario continuar los estudios de investigación a poblaciones de lactantes sanos o realizar seguimiento del desarrollo neuroconductual y así prevenir la aparición de la discapacidad motora e intelectual. Se sugiere que la prueba sea utilizada como tamizaje en el área de pediatría, con la participación de profesionales como terapeutas físicas, ocupacionales, nutricionistas y enfermeras, capacitados en el manejo de la prueba y de las actividades posteriores a la detección de alteraciones, con programas de atención temprana.

Se sugiere contar con protocolos de manejo en las áreas de atención y cuidado de los niños, en donde los padres sean continuamente informados e involucrados en la valoración y los avances en la atención y realizar la vigilancia en las actividades de estimulación que son más favorables para cada caso. 


\section{Referencias bibliográficas}

Alvarado, G. et al. (2013): "Modelo de vigilancia y seguimiento del neurodesarrollo infantil : experiencia en la Clínica de Medicina Familiar Tlalpan”. Rev Esp Médica Quirurgica, 18 (19): 19-30.

Bolaños, C. et al. (2015): "Indicadores de riesgo del Perfil de Conductas de Desarrollo como instrumento para la detección temprana de retrasos y alteraciones del desarrollo". Acta Pediátrica, 36: 135-146.

Bolaños, C. et al. (2006): "Validez de correlación del perfil de conductas de desarrollo con la escala de desarrollo infantil Bayley II”. Acta Pediátrica Mexico, 27 (4): 190-199.

Chávez, R. et al. (2012): “Secuelas de encefalopatía perinatal”. Revista Medica Instituto, 50 (2): 147-155.

Fernández Carrocera, L. A. et al. (1999): "El neurodesarrollo a los dos años de vida de neonatos tratados en una unidad de cuidados intensivos neonatales". Revista Panamericana de Salud Pública, 5 (1): 29-35.

González, R. R. et al. (2013): "Edad de presentacion de los reactivos del Test de Denver II en Ninos de 0 a 4 años de edad del Estado de Morelo". Salud Mental, 36 (6): 459-470.

Iceta, A. y Yoldi, M. E. (2002): "Psychomotor development of the child and its evaluation in primary care". Anales Del Sistema Sanitario de Navarra, 25 (Suppl 2): 35-43.

Insituto Nacional de Estadística, INEGI (2010): Principales resultados del Censo de Población y Vivienda 2010 (en línea). <http://www.inegi.org.mx/est/contenidos/proyectos/ccpv/cpv2010/iter_2010.aspx>, acceso 4 de abril de 2018.

Pelayo, H. et al. (2015): "Propuesta de prevención interactiva para bebés con factores de riesgo neurológico". Revista de Ciencias Clínicas, 14 (1): 21-29.

Salazar, A. et al. (2006): "Modificaciones de la escala de Denver en la evaluación de las condiciones del neurodesarrollo, en niños atendidos con hipoxia neonatal en una unidad de terapia intensiva". Revista Mexicana de Neurociencia, 7 (1): 88-99.

Salgado, P. (2007): Desarrollo Motor Normal, análisis desde el enfoque de neurodesarrollo (en línea). <https://www. santafe.gov.ar/index.php/educacion/content/download/149393/732110/file/Neurodesarrollo.pdf>, acceso 4 de abril de 2018.

Sánchez, C. et al. (2015): "El desarrollo del niño hasta los 12 meses. Orientaciones al pediatra para su vigilancia con la familia". Acta Pediátrica de México, 36 (6): 480-496.

Sánchez, C. et al. (2014): "La vigilancia del desarrollo en el primer año de vida”. Acta Pediatrica Mexico, 35: 356360.

Schapira, D. I. T. (2007): "Comentarios y aportes sobre desarrollo e inteligencia sensorio motriz en lactantes. Actualización bibliográfica”. Rev. Hosp. Mat. Inf. Ramón Sar Dá, 26 (1): 21-27. 